\title{
Investigación
}

\section{La gestión ambiental en el ordenamiento territorial para la conservación de los servicios ambientales en Rioblanco (Tolima) ${ }^{1}$}

\section{Environmental management in land-use planning for the conservation of environmental services in Rioblanco (Tolima)}

Pilar Andrea Martínez Manrique ${ }^{2}$

Carlos Humberto González Escobar ${ }^{3}$

Recepción: 17/05/2020 • Aprobación: 03/02/2021 • Publicación: 17/09/2021

Para citar este artículo

Martínez Manrique, P. A., \& González Escobar, C. H. (2021). La gestión ambiental en el ordenamiento territorial para la conservación de los servicios ambientales en Rioblanco (Tolima). Dos mil tres mil, 23, e235. https://doi.org/10.35707/dostresmil/23235

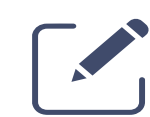

\section{(c) $(1)(\Theta \Theta$}

\footnotetext{
${ }^{1}$ Este artículo es resultado del proyecto de investigación "La gestión ambiental en el ordenamiento territorial para la conservación de los servicios ambientales en Rioblanco Tolima", realizado como requisito de grado por Pilar Andrea Martínez Manrique en la Maestría en Desarrollo Sostenible y Medio Ambiente de la Universidad de Manizales y con la asesoría del Ph. D. en Desarrollo Sostenible, Carlos Humberto González Escobar.

2 Candidata a Maestría en Desarrollo Sostenible y Medio Ambiente de la Universidad de Manizales, Correo electrónico: pilaranmm@gmail.com

${ }^{3}$ Maestría en Desarrollo Sostenible y Medio Ambiente de la Universidad de Manizales, Correo electrónico: redesom@umanizales.edu.co
} 
Resumen. La Constitución Política de 1991 contiene dentro de su espíritu la descentralización del Estado, al empoderar los territorios y determinar que son los responsables de ordenar y regular su uso desde lo productivo, social y ambiental; en el año 1997 se expide la Ley 388 que reglamenta todos los aspectos relacionados con el ordenamiento territorial.

El objetivo de este trabajo es analizar la gestión ambiental dentro del ordenamiento territorial para la conservación de los servicios ecosistémicos en el municipio de Rioblanco (Tolima). Para ello, se analizaron instrumentos de planificación del territorio, se entrevistaron actores sociales, institucionales y comunitarios y por último se realizó un análisis de los factores que afectan la conservación de los servicios ecosistémicos. Como resultado, se observa desarticulación entre los instrumentos de planificación, lo cual genera un desarrollo que no responde a las necesidades reales del territorio. Rioblanco cuenta con tres (3) áreas protegidas reconocidas por el Sistema Nacional de Áreas Protegidas (sINAP), complejos de páramos y ecosistemas con alta importancia ambiental y baja representatividad en el sinAp, pero que no se ven reflejados en los instrumentos de ordenación y planificación; también se logró identificar que las comunidades y autoridades ambientales no se sienten partícipes en la formulación de estos instrumentos, distanciándolos en la contribución al desarrollo de la región; por último, se logró determinar que el Municipio es un territorio que se destaca en lo sectorial, especialmente en la minería, lo cual puede afectar su estructura ecológica, sumado a que carece de escenarios para la protección del territorio.

Abstract. The 1991 Political Constitution contains within its spirit the decentralization of the State, by empowering the territories and determining that are responsible for ordering and regulating their use from the productive, social and environmental aspects. For this reason, in 1997 Act 388 was issued, which regulates all aspects related to land-use planning.

The objective of this work is to analyze environmental management within the territorial ordering for the conservation of ecosystem services in the municipality of Rioblanco (Tolima). To this effect, territorial planning instruments were analyzed; social, institutional and community actors were interviewed; and finally, an analysis of the factors that affect the conservation of ecosystem services was carried out. As a result, there is a lack of articulation among planning instruments, which generates a development that does not respond to the real 
needs of the territory. Rioblanco has three (3) protected areas recognized by the National System of Protected Areas (SINAP), paramos and ecosystems complexes with high environmental importance and low representation in SINAP, but which are not reflected in the management and planning instruments. It was also possible to identify that the communities and environmental authorities do not feel involved in the formulation of these instruments, distancing them from contributing to the development of the region. Lastly, it was determined that the Municipality is a territory that stands out in the sector, especially in mining, which can affect its ecological structure, added to the fact that it lacks scenarios for the protection of the territory.

\section{Palabras claves}

Desarrollo territorial, ordenamiento territorial, gestión ambiental, conservación.

Key words

Territorial development, land-use planning, environmental management, conservation. 


\section{Introducción}

Al tener en cuenta el orden jurídico colombiano, en el que la Constitución Política de 1991 es considerada la norma de normas y contiene dentro de su espíritu la descentralización del Estado, al empoderar los territorios y determinar que son los responsables de ordenar y regular su uso desde lo productivo, social y ambiental, en el año 1997 se expide la Ley 388 que reglamenta y define todos los aspectos relacionados con el ordenamiento territorial.

Dicha Ley les proporciona a los municipios la autonomía para ordenar y reglamentar el uso del suelo en sus territorios. Para esto, se crean tres instrumentos de planificación de acuerdo con el número de habitantes del Municipio, estos son: Planes de Ordenamiento Territorial (РОт) para municipios con población superior a 100000 habitantes, Planes Básicos de Ordenamiento Territorial (Рвот) para municipios entre los 30000 y 100000 habitantes y Esquemas de Ordenamiento Territorial (ЕОт) para municipios con población inferior a 30000 habitantes.

Por otra parte, las determinantes ambientales son un insumo importante para lograr la articulación de los procesos de revisión, ajuste y reformulación de los Planes de Ordenamiento Territorial con las propuestas de ordenamiento ambiental promovidas desde los ámbitos regionales y nacionales, como lo son las áreas protegidas, los Planes de Ordenación Forestal, Planes de Manejo de Páramos y Humedales, los Planes de Ordenación y Manejo de Cuencas y demás instrumentos de planificación existentes.

En particular, el municipio de Rioblanco cuenta con una población aproximada de 24345 habitantes, por ello su planificación territorial se realizó a través de un Plan Básico de Ordenamiento Territorial (Рвот) formulado en el año 2000 y con una vigencia de 12 años (3 periodos administrativos) y que, con todas las dinámicas actuales del territorio, se encuentra desactualizado y técnicamente se hace necesaria la revisión, ajuste, actualización e inclusión de la estructura ecológica principal, para identificar áreas de importancia ambiental para el Municipio.

La problemática central identificada en este trabajo radica en que en el ordenamiento territorial del municipio de Rioblanco no se incluyen aspectos relacionados con la gestión y conservación de servicios ecosistémicos, que son definidos como:

Los beneficios directos e indirectos que la humanidad recibe de la biodiversidad y que son el resultado de la interacción entre los diferentes componentes, estructuras y funciones que constituyen la biodiversidad. De acuerdo con la propuesta de Ecosistemas del Milenio, los servicios ambientales ecosistémicos son los beneficios que obtienen las personas de los ecosistemas. Estos incluyen los servicios de suministro, regulación y culturales que directamente afectan a las personas, además de los servicios necesarios para mantener los procesos ecológicos. (Convención de Ramsar y Grupo de Contacto eHAA, 2008, s. p.).

La inclusión de objetivos, programas y líneas estratégicas relacionados con la conservación de la biodiversidad es fundamental en el territorio, ya que es a través de esta biodiversidad que se genera la provisión de servicios ecosistémicos esenciales para la sostenibilidad del territorio. 
El objetivo de este trabajo es realizar un análisis que permita identificar la gestión ambiental que se desarrolla en el ámbito municipal, para la conservación de los servicios ecosistémicos a través del ordenamiento del territorio en el municipio de Rioblanco, Tolima.

\section{Metodología}

Para fines de este trabajo, el área de estudio fue el municipio de Rioblanco, localizado en el sur del departamento del Tolima, con coordenadas geográficas céntricas $03^{\circ} 32^{\prime 2} 20^{\prime \prime}$ latitud norte y $75^{\circ} 39^{\prime} 30^{\prime \prime}$ longitud oeste, se encuentra a una altura promedio de $970 \mathrm{msnm}$ y tiene una extensión promedio de 204944 hectáreas (Figura 1).

El tipo de investigación es proyectiva, haciendo énfasis en lo descriptivo y analítico, bajo la perspectiva documental, por lo que parte del análisis teórico y cartográfico de la información, se complementa a través del uso de información secundaria y de esta manera llegar a la comprobación de la pregunta de investigación planteada.

Figura 1. Área de estudio

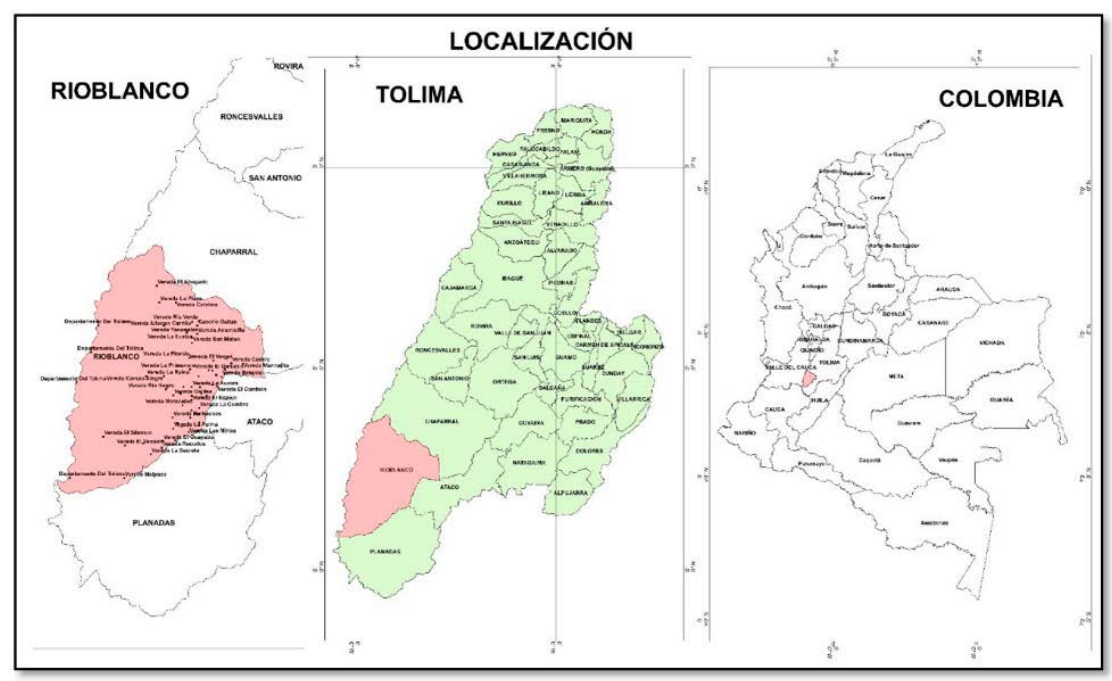

Fuente: IGAC, 2019, citado por Martínez P., 2020, p. 35

El desarrollo metodológico consistió en lo siguiente:

- Se realizaron seis (6) entrevistas a tres tipos de actores sociales diferentes, entre los que se encuentran las autoridades ambientales, municipales y la sociedad civil. Se les preguntó sobre a) conocimiento en temas de ordenamiento territorial, b) el tipo de participación que tienen en la toma de decisiones frente al ordenamiento territorial, y c) cómo perciben la articulación de los instrumentos de planificación: Plan Básico de Ordenamiento Territorial (рвот) у el Plan de Desarrollo. 
- Se analizó la información cartográfica temática disponible relacionada con: Áreas del Sistema Nacional de Áreas Protegidas (sINAP) y otras estrategias de conservación (no reconocidas como áreas protegidas, pero que cumplen un papel fundamental en la provisión de servicios ecosistémicos), coberturas de la tierra; ecosistemas estratégicos; prioridades de conservación y la información sectorial de títulos mineros e hidrocarburos.

- Se elaboró un análisis de los instrumentos de planificación: Plan de Desarrollo 2016-2019 y el Plan Básico de Ordenamiento Territorial del municipio de Rioblanco, tomando como referencia los aspectos relacionados con conservación de los servicios ecosistémicos en el Municipio.

\section{Resultados}

\subsection{Conocimiento en cuanto a políticas de ordenamiento: Articulación de los instrumentos de planificación}

En el caso del ente territorial (Alcaldía Municipal de Rioblanco), si bien los funcionarios conocen sobre la normatividad, se observó que la Secretaría de Desarrollo Económico y Ambiental no tiene suficiente incidencia sobre la toma de decisiones de planificación territorial. Esto puede deberse a que la coordinación tanto de la elaboración como de la ejecución de proyectos de los planes de desarrollo y el Plan Básico de Ordenamiento Territorial (Рвот), dependen directamente de la oficina de Planeación Municipal. En este sentido, es importante que desde la Secretaría de Planeación se incorporen las directrices de determinantes ambientales de la autoridad ambiental y estas se articulen con los proyectos sectoriales que se tengan propuestos, para evitar los conflictos de uso de suelo en el territorio.

Desde el punto de vista de las comunidades, no existen espacios de participación social a través de los cuales se puedan canalizar las necesidades, las expectativas, los valores, sentidos y significados con respecto al territorio, así como tampoco las relaciones de la sociedad y la naturaleza. Al no existir canales de participación real y efectiva, se frustran las posibilidades de interacción, el diálogo abierto, en exponer la voz ciudadana ante los escenarios y espacios institucionales, y que de esta manera sus planteamientos, visiones y enfoques, se incorporen a los procesos de planificación y gestión de desarrollo sustentable del territorio; haciéndose de manera efectiva mediante el Consejo de Planeación Territorial (СРT), que representa a la sociedad civil en la planeación del desarrollo, por mandato de la Constitución Política de Colombia de 1991, en su artículo 340, y las Leyes 152 de 1994 y 388 de 1997, compiladas por el Decreto 3600 de 2007 y la Ley 1454 de 2011.

Si bien, las autoridades ambientales son conocedoras de la normativa referente a la inclusión de aspectos ambientales en los instrumentos de planificación territorial y son las que dictan las directrices sobre determinantes ambientales para el ordenamiento territorial, para el caso del municipio de Rioblanco, la corporación de la región, Cortolima, no se articula de 
manera adecuada con la Secretaría de Planeación para incluir el eje ambiental en el ordenamiento del territorio.

\subsection{Apropiación del tema ambiental}

En el municipio de Rioblanco es evidente que la toma de decisiones en la construcción y actualización del Plan Básico de Ordenamiento Territorial (рвот) depende de terceros, debido a la contratación de consultores para su realización; en este sentido, se observó una debilidad estructural en la Alcaldía, que tiene que ver con la falta de apropiación presupuestal para la actualización del Plan Básico de Ordenamiento Territorial (Рвот), por lo cual se dificulta realizar espacios de participación de otras dependencias e instituciones en la toma de decisiones sobre dicho plan.

Ninguna de las personas entrevistadas conoce los términos de estructura ecológica, determinantes ambientales, y demás conceptos del tema ambiental inmersos en el Plan Básico de Ordenamiento Territorial (Рвот). Sin embargo, sí coinciden con los actores institucionales en que se deben incluir las microcuencas que abastecen acueductos, los ríos como actores de derecho y los páramos que están fuera de los Parques Nacionales Naturales de Colombia.

La dificultad presupuestal del Plan Básico de Ordenamiento Territorial (Рвот) impide llegar a todos los actores comunitarios. Por ende, obstaculiza las propuestas de actores locales de veredas y corregimientos, insumos que nutren los instrumentos de planificación del territorio a partir de una visión clara y real del Municipio, y que podrían generar soluciones de fondo a las problemáticas ambientales.

Es un requisito de procedimiento para la adopción del Plan Básico de Ordenamiento Territorial (Рвот), la inclusión de los diferentes actores tanto comunitarios como de otros sectores; es por ello que, con el fin de lograr esta participación, se crearon los Consejos de Planeación Territorial (СРT), que se constituyen como representantes de la sociedad civil en la planeación del desarrollo territorial. En este contexto, si las comunidades y la sociedad civil en general no se ven reflejadas o incluidas en la construcción del Plan Básico de Ordenamiento Territorial (Рвот), se evidencia un deficiente funcionamiento del Consejo de Planeación Territorial del municipio de Rioblanco.

Tanto Cortolima como Parques Nacionales Naturales de Colombia, al ser las autoridades ambientales con jurisdicción en el territorio, conocen los conceptos y aplicabilidad sobre la inclusión de aspectos ambientales en el Plan Básico de Ordenamiento Territorial y por obligación deben establecer las directrices frente a determinantes ambientales para la actualización del mismo. En este sentido, se puede inferir que también hay una debilidad en Cortolima, en primera instancia, porque el Plan Básico de Ordenamiento Territorial (Рвот) antes de ser aprobado por el Concejo Municipal, pasa por la revisión y concertación con la autoridad ambiental; en esta concertación se define qué se debe o no incluir en términos ambientales; es decir, que si la actualización del Plan Básico de Ordenamiento Territorial (Рвот) de Rioblanco no identifica 
los aspectos ambientales, es porque no hubo una revisión concienzuda y a profundidad del documento por parte de Cortolima.

\subsection{Participación ciudadana}

De manera abierta, los funcionarios del Municipio expresan que los mecanismos de participación en la construcción del Plan de Desarrollo y en el Plan Básico de Ordenamiento Territorial (Рвот) son ineficientes, pocas reuniones que se convierten en la lista de mercado y en la solicitud de pequeños proyectos locales (puentes, viviendas, vías, entre otras), sin incluir los ítems ambientales que debe contener cualquier proyecto.

No se tiene información de organizaciones comunitarias, más allá de las Juntas de Acción Comunal (JAC) y en algunas asociaciones de productores, no existe una cultura de asociatividad frente al tema ambiental de organizaciones que encarnen la defensa del tema ambiental desde lo comunitario.

Las comunidades coinciden en que la participación en los procesos de construcción de los documentos de planificación y ordenación ha sido muy baja, las administraciones municipales realizan pocas reuniones con las comunidades, en las que se concentran en temas de infraestructura y se deja por fuera lo ambiental, se firman listados de asistencia que validan la participación y con esto se concluye dicha participación; la visión de desarrollo que contienen estos documentos obedecen al imaginario de los contratistas o consultores que se encargan de elaborar los documentos, dando una señal que se construyeron de manera participativa, pero esto está muy alejado de la realidad.

Como autoridades ambientales no existe una participación de la construcción de estos documentos de ordenación y planificación del territorio, principalmente porque desde las administraciones municipales no se convocan.

Dentro de los análisis de la información recolectada, es importante resaltar en términos de participación, que no existen escenarios que permitan que las comunidades y las autoridades ambientales participen de manera activa, desde la administración municipal se contratan consultorías que se encargan de estructurar los documentos con una baja participación y visión comunitaria e interinstitucional.

\subsection{Análisis de información cartográfica temática}

Como resultado del análisis de la información cartográfica disponible para el área de estudio se obtuvo que el municipio de Rioblanco está conformado por 18 coberturas de tierra, donde las coberturas naturales predominan con un total de 147818 hectáreas correspondientes al $72 \%$ del área del Municipio, mientras que las coberturas trasformadas ocupan el $28 \%$ del territorio. Se observó que las coberturas con mayor extensión fueron: bosques densos y herbazales con 76122 hectáreas y 44042 hectáreas respectivamente (Figura 2). 
Figura 2. Coberturas de la tierra

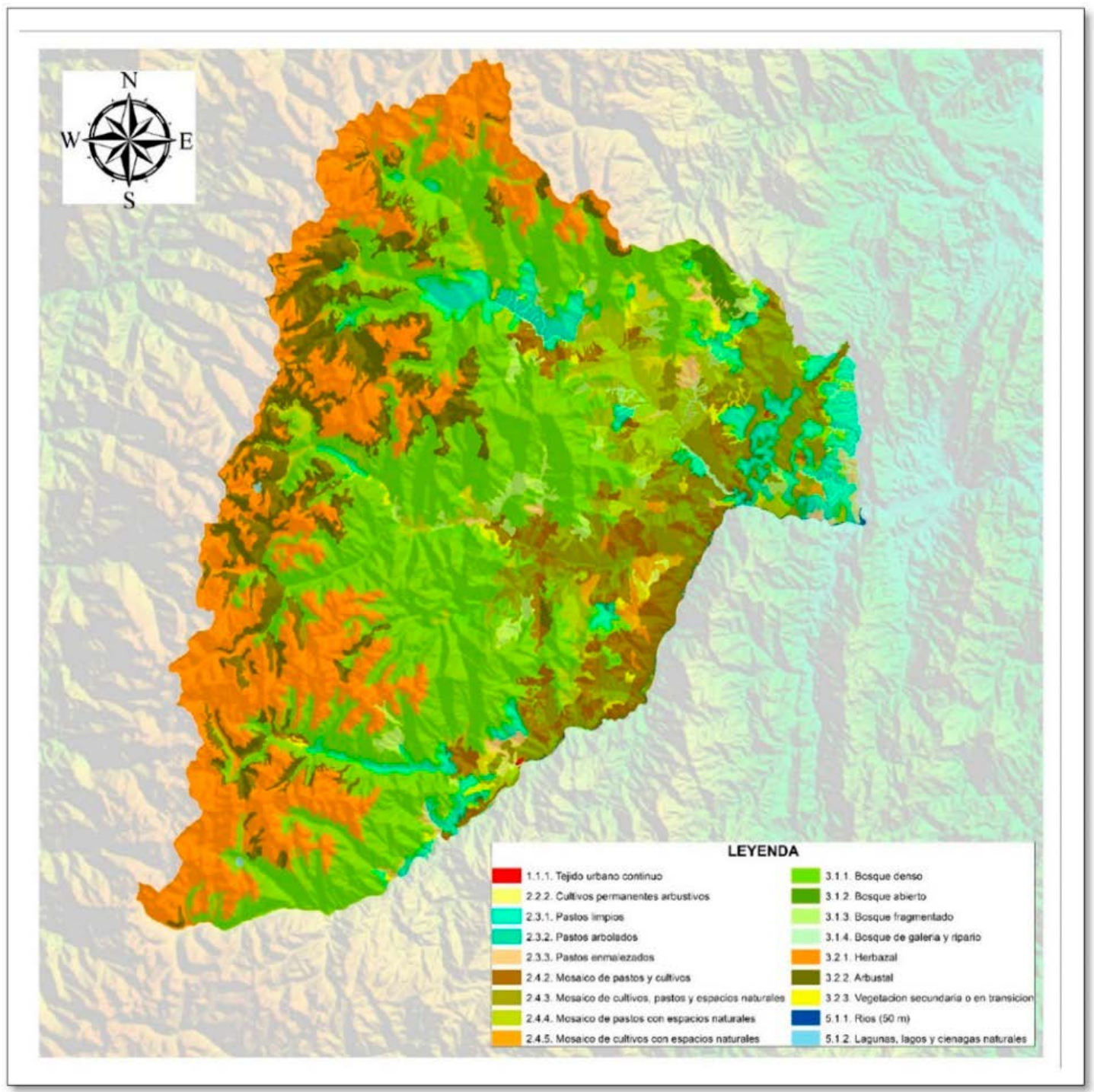

Fuente: IDEAM, 2012, citado por Martínez P., 2020, p. 54

La tendencia de las coberturas identificadas obedece a la presencia de tres (3) áreas protegidas del Sistema Nacional de Áreas Protegidas (sINAP), que corresponden al parque natural regional We'Pe' Walla y los parques nacionales naturales Nevado del Huila y Las Hermosas; este último con un 45,72\% de su área dentro del municipio de Rioblanco (Figura 3). 
Figura 3. Áreas protegidas Sistema Nacional de Áreas Protegidas (sINAP)

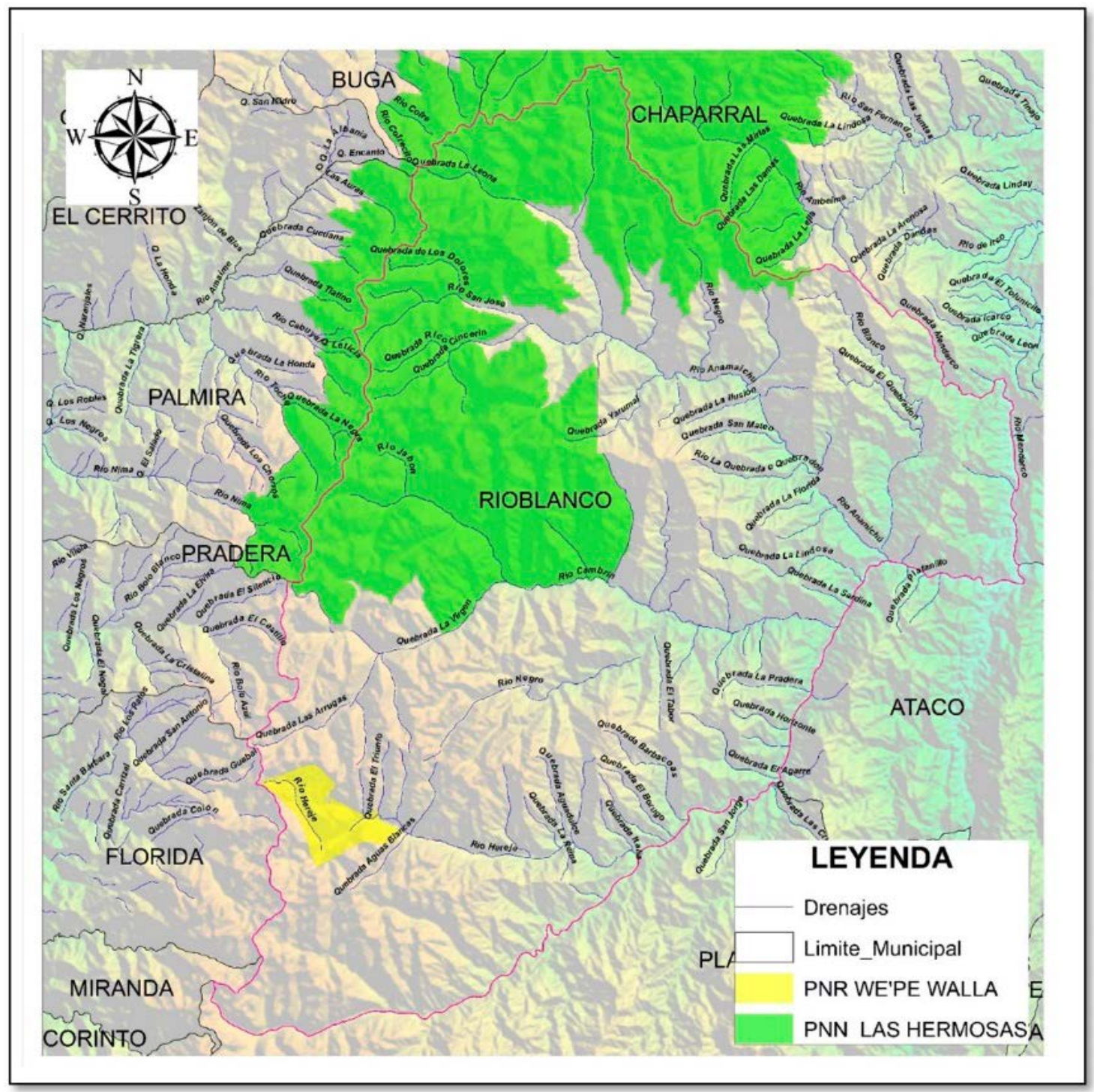

Fuente: RUNAP, 2020, citado por Martínez P., 2020, p. 52

Asociado a estas áreas protegidas, se identificó la presencia de ecosistemas estratégicos, principalmente representados por ecosistemas de páramos, distribuidos en dos tipos de complejos que son: complejos de páramos Las Hermosas y Nevado de Huila-Moras con 48241 hectáreas y 731 hectáreas, respectivamente (Figura 4). 
Figura 4. Complejos de Páramos

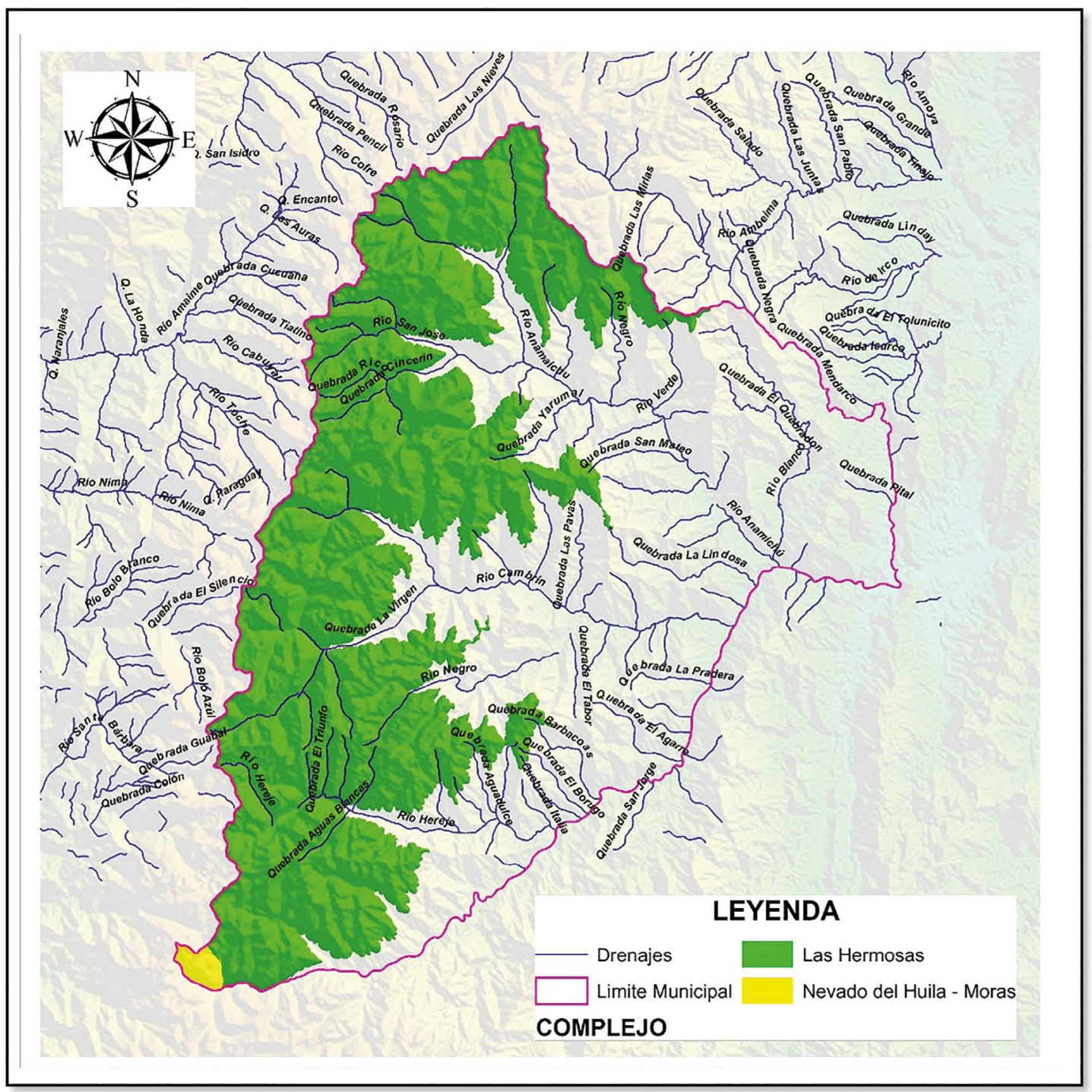

Fuente: Instituto Alexander Von Humboldt, 2011, citado por Martínez P., 2020, p. 56

Otro aspecto importante, desde el punto de vista biofísico y ecosistémico identificado, fue la presencia de cuerpos de agua que nacen en los páramos y bosques altos andinos, estos son: Anamichú, Cambrín, Saldaña, Hereje y Rioblanco (Figura 5); todos recorren lo largo y ancho del Municipio y a su vez abastecen acueductos, lo cual resalta la importancia de los ecosistemas, al ser provisión de servicios ecosistémicos relacionados con el abastecimiento de recursos hídricos para consumo humano. 
Figura 5. Recurso hídrico

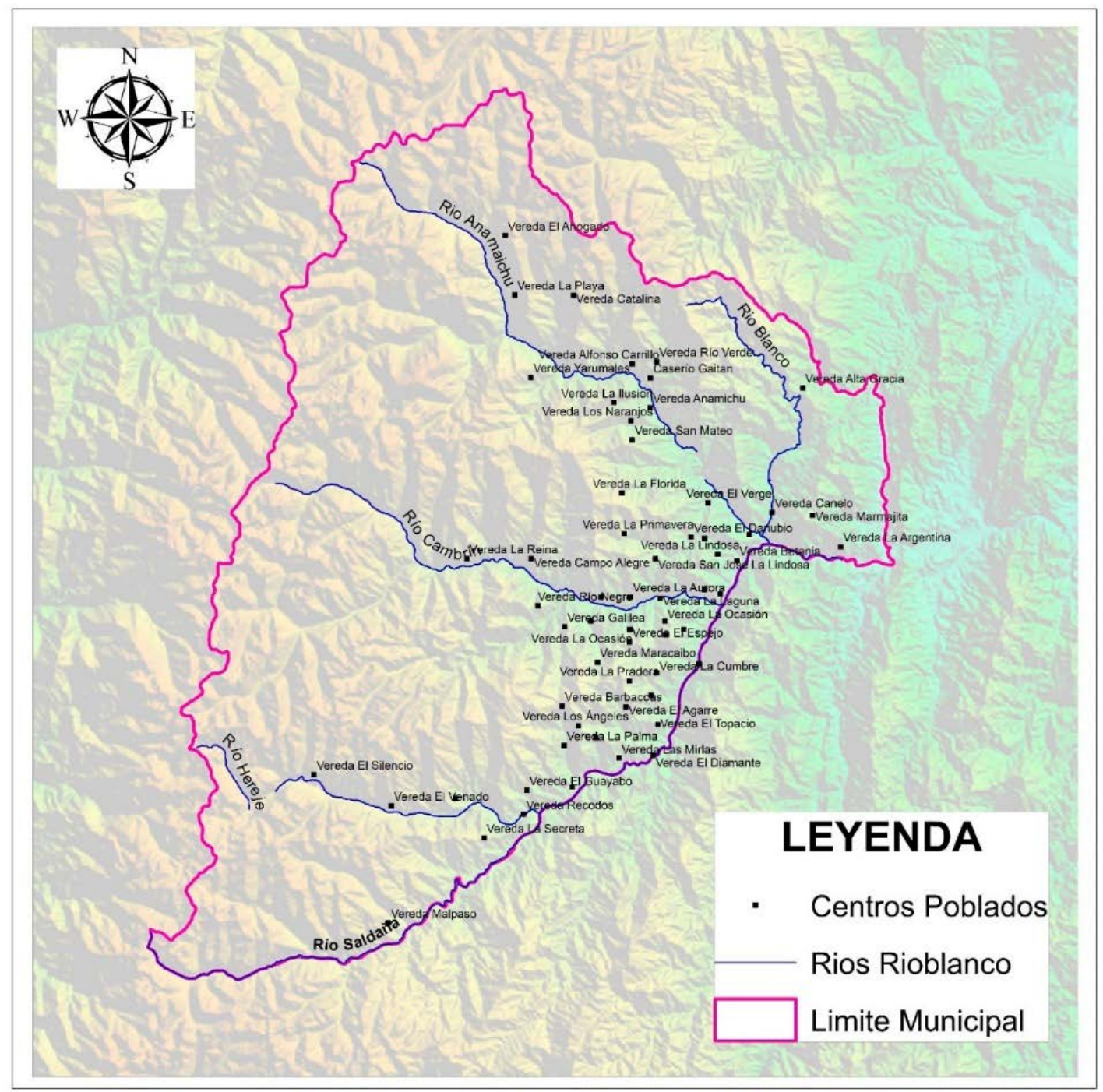

Fuente: IGAC, 2019, citado por Martínez P., 2020, p. 57

Con base al estudio de prioridades de conservación, definidas por Andrade \& Corzo (2011), se identificó para el Municipio la prioridad baja insuficiencia y urgente (Figura 6), que ocupa una extensión de 27410 hectáreas, definida como ecosistemas con baja representatividad en el Sistema Nacional de Áreas Protegidas y con una prioridad de conservación urgente, debido a la disminución en área y altas presiones a las que están sometidos, por la ampliación de la frontera agrícola y la implementación de proyectos sectoriales. 
Figura 6. Prioridades de conservación nacional

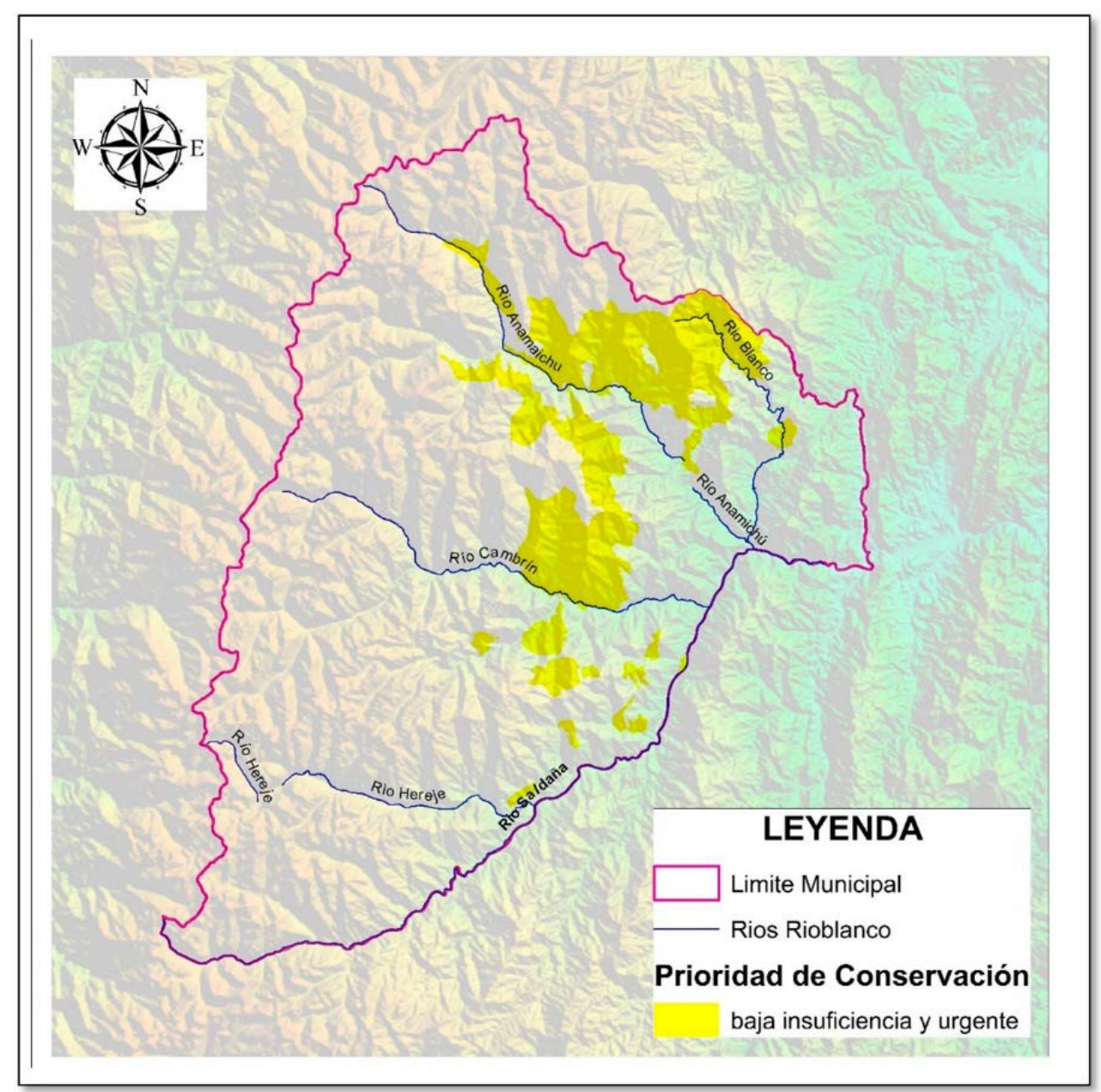

Fuente: Andrade \& Corzo, 2011, citado por Martínez P., 2020, p. 60

En contraste, con la información cartográfica temática ambiental, se identificaron proyectos sectoriales enfocados en exploración y explotación de minerales e hidrocarburos; en el municipio de Rioblanco, según datos de la Agencia Nacional de Minería (2019), se identificaron un total de 20915 hectáreas en títulos mineros, distribuidos en 8 títulos (Tabla 1; Figura 7). El título de mayor extensión corresponde al código HJD-12221 con 4903 hectáreas, para la extracción de cobre, oro plata y sus derivados, y el título de menor extensión corresponde al código HC3152 con 22 hectáreas, igual para la extracción de oro. En cuanto al sector de hidrocarburos se encontró un total de dos bloques de hidrocarburos de la clase disponibles; es decir, yacimientos identificados que no han sido asignados para exploración ni explotación. Cubren un área total 
de 54267 hectáreas y el operador es la Agencia Nacional de Hidrocarburos (ANH). Frente al tema sectorial, el Municipio pierde total autonomía en la determinación de dichas áreas, esta decisión pasa por el orden nacional que es poco consultivo con lo local y regional en su delimitación y otorgamiento.

Figura 7. Títulos mineros

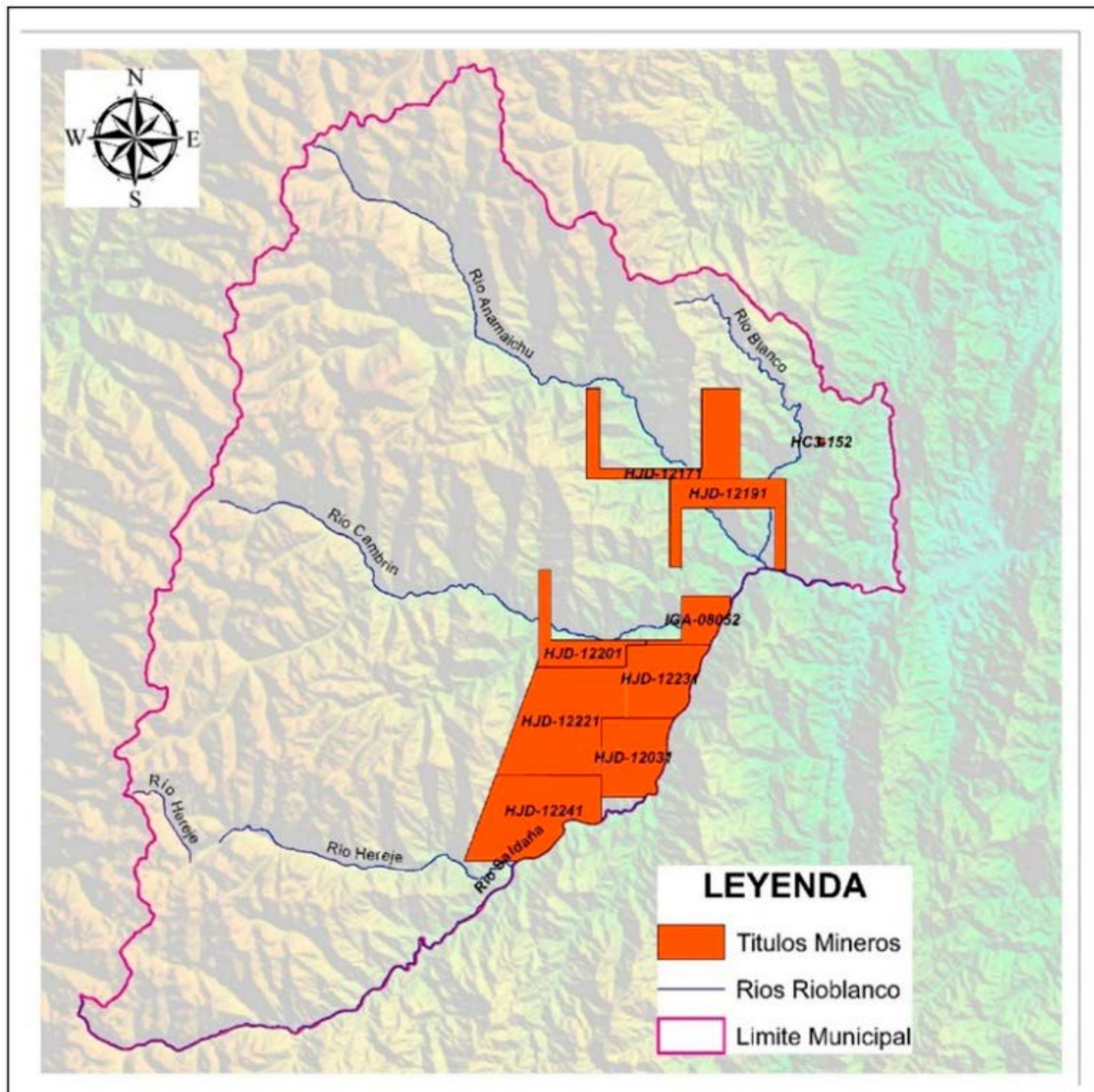

Fuente: Agencia Nacional de Minería, 2019, citado por Martínez P., 2020, p. 70 
Tabla 1. Títulos mineros

\begin{tabular}{|c|c|r|}
\hline ID & Código título & Área (HAS) \\
\hline 2 & IGA-08052 & 1.005 \\
\hline 3 & HJD-12201 & 1.517 \\
\hline 4 & HJD-12191 & 2.226 \\
\hline 5 & HJD-12031 & 2.302 \\
\hline 6 & HJD-12231 & 2.322 \\
\hline 7 & HJD-12171 & 2.643 \\
\hline 8 & HJD-12241 & 3.975 \\
\hline 9 & HJD-12221 & 4.903 \\
\hline & Total & 20.915 \\
\hline
\end{tabular}

Fuente: Agencia Nacional de Minería, 2019, p. 70

Figura 8. Bloques de hidrocarburo

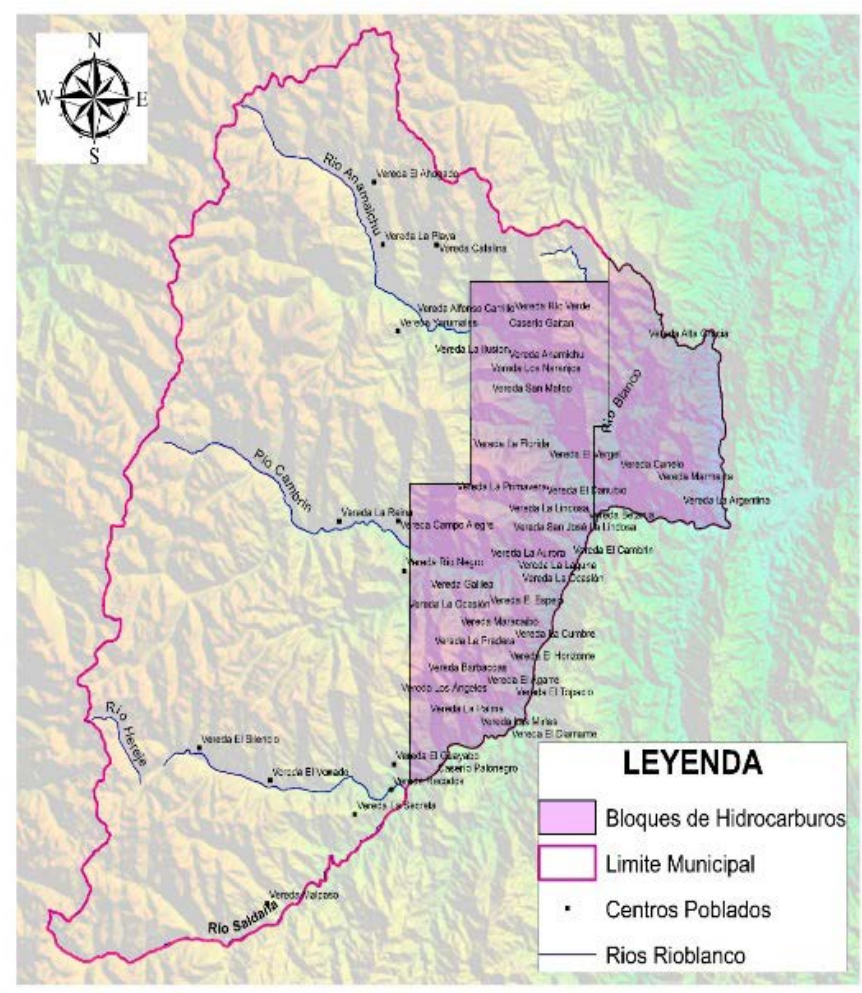

Fuente: Agencia Nacional de Hidrocarburos, 2019, citado por Martínez P., 2020, p. 71 


\section{Análisis de instrumentos de planificación}

En el análisis de los instrumentos de planificación territorial, Plan de Desarrollo Municipal y Plan Básico de Ordenamiento Territorial (РвОт), se encontraron algunos aspectos relacionados con la conservación ambiental, pero no directamente tienen que ver con la gestión para la conservación de servicios ecosistémicos.

\subsection{Plan Básico de Ordenamiento Territorial}

Al municipio de Rioblanco, por su cantidad de habitantes, le corresponde formular un Plan Básico de Ordenamiento Territorial (Рвот) conforme a lo dispuesto en la Ley 388 de 1997. En el año 2000, el Municipio formula su Plan Básico de Ordenamiento Territorial (Рвот), que está conformado por tres componentes: diagnóstico, formulación y plan de inversiones. El componente de formulación contempla la clasificación del territorio en tres tipos de suelo: suelo urbano, suelo de expansión urbana y suelo rural. Se realizó el análisis de cada uno de los tres tipos de suelo, para ver qué contemplan en aspectos ambientales y servicios ecosistémicos.

En este orden de ideas, se identificó que el suelo urbano del municipio de Rioblanco está clasificado en áreas de conservación y protección de los recursos naturales urbanos; zonas de reserva forestal; zonas de protección hídrica; parque ecológico principal, que comprende zonas verdes del área urbana, las zonas de ladera, cerros que encierran el área urbana, las áreas de protección de la quebrada Arrastradero y río Rioblanco respectivamente.

En cuanto al suelo rural se identificó la siguiente clasificación: áreas de especial significancia ambiental, conformadas por los parques nacionales naturales Las Hermosas y Nevado del Huila y sus áreas amortiguadoras; área forestal protectora, que incluye las áreas con pendientes mayores al $50 \%$; áreas de rondas de cuerpos de agua, que comprenden franjas de suelo ubicados paralelamente a los cauces de agua o en la periferia de los nacimientos o cuerpos de agua; áreas de riesgo natural o áreas de riesgo hídrico, que son áreas expuestas a crecientes y desbordamientos de cauces de los ríos.

\subsection{Plan de Desarrollo Municipal "Todos Somos Rioblanco" 2016-2019}

El Plan de Desarrollo del municipio de Rioblanco fue aprobado bajo acuerdo municipal número 10 de 2016 "Por el cual se adopta el Plan de Desarrollo para el municipio de Rioblanco Tolima 2016-2019”. En el artículo 5 del acuerdo se adoptan los cinco (5) ejes o pilares que conforman la estructura del Plan de Desarrollo, sobre la cual se desarrollan los programas de gobierno municipal. Estos cinco ejes son: 1) equidad social, 2) competitividad agropecuaria, 3) paz y desarrollo social, 4) desarrollo urbano y ordenamiento territorial y, por último, 5) prácticas de buen gobierno.

Dentro del análisis que se realizó del Plan de Desarrollo del municipio de Rioblanco se obtuvo como resultado que, de los cinco ejes que lo conforman, solo dos ejes tienen relación con la gestión para la conservación de la biodiversidad y servicios ecosistémicos. Estos ejes son: 
- Eje 1: Equidad Social. Este eje tiene como principio producir un cambio en la calidad de vida en los aspectos social, económico, cultural y ambiental, en este eje se encuentra el sector "Agua potable y saneamiento básico". Este sector contempla el programa "Todos con servicios públicos de calidad y cobertura", cuyo objetivo es: "Optimizar la calidad del agua apta para el consumo humano en el municipio de Rioblanco"; en este contexto, el eje promueve la gestión relacionada con la conservación de servicios ecosistémicos de abastecimiento en relación con el suministro de agua potable para los habitantes del Municipio.

- Eje 3: Competitividad agropecuaria. Este eje tiene como premisa el fortalecimiento del sector rural en lo productivo, para posesionar económicamente al Municipio en lo regional y nacional. Dentro de este eje se encuentra el sector "Ambiental", que comprende un programa denominado: "Por un medio ambiente con desarrollo sostenible para todos", que en dos de sus objetivos plantea "reducir el deterioro ambiental del municipio" y "garantizar la conservación de los ecosistemas, la biodiversidad, sus servicios ecosistémicos y los procesos hidrológicos de los que depende la oferta agua". Este último, muy relacionado con los servicios ecosistémicos de regulación y abastecimiento de recurso hídrico. Este mismo objetivo está incluido en el programa "Todos por la adaptación y mitigación del cambio climático en Rioblanco".

\section{Discusión}

Los resultados de las encuestas sugieren principalmente la falta de articulación entre la entidad territorial (Alcaldía Municipal) y los diferentes actores, tanto institucionales como agremiaciones y sociedad civil, en la formulación y puesta en marcha de los instrumentos de planificación del territorio, desconociendo la importancia de los aportes en la visión del Municipio. La participación efectiva de la comunidad se presenta a través del Consejo de Planeación Territorial (СРТ), que representa a la sociedad civil en la planeación del desarrollo, por mandato de la Constitución Política de Colombia de 1991, en su artículo 340, y las Leyes 152 de 1994 y 388 de 1997.

En términos biofísicos, se puede decir que gran parte del municipio de Rioblanco se encuentra sobre coberturas naturales ( $72 \%$ en coberturas naturales), las cuales están asociadas principalmente al Parque Nacional Natural Nevado del Huila, Parque Nacional Natural Las Hermosas y Parque Natural Regional Páramo del Meridiano We'Pe Walla, siendo las principales áreas de provisión de servicios ecosistémicos del territorio de Rioblanco, especialmente los asociados al recurso hídrico y que cuentan con un blindaje jurídico para su protección, siendo estas reconocidas como áreas protegidas del Sistema Nacional de Áreas Protegidas (sINAP). Estas potencialidades ambientales no cuentan con un análisis profundo dentro del instrumento de ordenación (Plan Básico de Ordenamiento Territorial), y son incluidas como un acápite del documento sin profundizar en su manejo y articulación con escenarios sociales y productivos. Se debe construir una estructura ecológica principal para el Municipio, como soporte técnico 
del Plan Básico de Ordenamiento Territorial (Рвот), que incluya estas áreas protegidas y otras prioridades de conservación municipal que permitan:

Garantizar el mantenimiento de la integridad de la biodiversidad, la provisión de servicios ambientales (agua, suelos, recursos biológicos y clima), como medida para garantizar la satisfacción de las necesidades básicas de los habitantes y la perpetuación de la vida. (Van Der Hammen \& G. Andrade, 2003, p. 21).

La información sectorial nos permite una mirada muy general de los intereses que tienen las entidades nacionales competentes en esta materia sobre el territorio municipal; en primer lugar, se obtuvo que el 10,21 \% (20915 hectáreas) tienen concesiones mineras. Si bien estos títulos mineros no presentan licencia de exploración ni explotación, tienen una vigencia de 30 años y en cualquier momento se puede iniciar la fase de exploración sobre estos territorios. Con los resultados obtenidos, no se evidencia que se presente un conflicto de estos títulos mineros con las áreas protegidas y ecosistemas estratégicos del Municipio. El conflicto ambiental-minero en Colombia se confirma cada día más, se estima que hay 571 títulos mineros en 203000 hectáreas donde no se pueden desarrollar proyectos mineros (Fierro et al., 2012). Para el año 2010, el tema de solicitudes mineras en el país era muy preocupante, debido a que más de la mitad de los páramos, las reservas forestales protectoras y los humedales Ramsar se encontraban con solicitudes, lo cual llevó a su exclusión en la modificación del Código de Minas (Ley 1382 de 2010).

Otro aspecto de la presión minera sobre el territorio (Rudas, 2010) establece que la actividad minera y particularmente la minería de superficie a cielo abierto, es catalogada como una actividad de alto riesgo para los ecosistemas y para la salud humana. Por ello se debe tener en cuenta estas afectaciones a la hora de establecer los usos del suelo que, desde el Plan Básico de Ordenamiento Territorial (Рвот), permitan regular o controlar la minería en el municipio de Rioblanco.

Por otra parte, el sector hidrocarburos no debe generar mayor preocupación, si bien se identificaron dos bloques de tipo disponible, estos no están concesionados a ninguna empresa. Según la definición del Código de Minas: "Las áreas disponibles de hidrocarburos, se constituyen como zonas identificadas con potencial, pero que aún no han sido definidas como yacimientos finales de exploración ni explotación" (Ley 685 de 2001).

Es importante relacionar esta información sectorial con las prioridades de conservación de la nación, por lo que en el municipio de Rioblanco se identificaron 87656 hectáreas que corresponde al $44 \%$ del área municipal como áreas prioritarias para la conservación de tipo baja insuficiencia y urgente. Según Andrade \& Corzo (2011), estas áreas que están poco representadas en el Sistema Nacional de Áreas Protegidas (SINAP) están siendo amenazadas por proyectos de desarrollo sectorial. Con la información cartográfica se pudo determinar que estas prioridades coinciden con los títulos mineros y bloques de hidrocarburos identificados en el territorio. 
Los instrumentos de planificación propios del Municipio, como son el Plan Básico de Ordenamiento Territorial (Рвот) у Plan de Desarrollo, a pesar de que incorporan aspectos ambientales en su contenido, no contemplan de manera directa la gestión ambiental relacionada con Servicios Ecosistémicos, siendo estos de gran importancia para el desarrollo de los territorios, tal como lo proponen Paruelo et al. (2015) en su trabajo Plan operativo para incorporar los servicios ecosistémicos en el ordenamiento territorial, en el que concluyen que las decisiones que se tomen sobre el ordenamiento del territorio influyen directamente en el flujo de bienes y servicios ecosistémicos.

En términos más específicos, en el Plan Básico de Ordenamiento Territorial (Рвот) del municipio de Rioblanco no se observó una Estructura Ecológica Principal (EEP) definida, esto puede deberse a que la reglamentación para incluir dicho concepto en los planes de ordenamiento de Colombia surgió en el año 2007, y el del municipio de Rioblanco se formuló en el año 2000. Es importante que el Municipio, aprovechando que el Plan Básico de Ordenamiento Territorial (Рвот) perdió vigencia, establezca este concepto en el nuevo instrumento, al considerar que la Estructura Ecológica Principal plantea que los elementos bióticos y abióticos son el soporte para el desarrollo de los territorios (Van der Hammen, 2003).

\section{Conclusiones y recomendaciones}

- Aproximadamente el $47 \%$ del suelo del Municipio son coberturas naturales y están bajo protección con la figura del parque nacional natural Las Hermosas, Nevado del Huila y el parque natural regional de Meridiano; se recomienda utilizar el análisis de prioridades de conservación que se identificó para ampliar estas áreas de protección, con el fin de garantizar la sostenibilidad del territorio en la parte baja y media de las cuencas Anamichú y Saldaña.

- Se recomienda al Municipio generar espacios de articulación interinstitucional con las autoridades ambientales, con el fin de definir usos de producción sostenible y estrategias de conservación de suelo sobre las zonas con función amortiguadora de las tres áreas protegidas, de tal manera que mitiguen las presiones y se evite la pérdida de cobertura en estos territorios.

- La construcción de una estructura ecológica principal, como soporte técnico de la ordenación del territorio, debe ser prioridad para el municipio de Rioblanco, lo cual permitirá determinar los elementos ambientales soportes para el desarrollo del territorio e incluirlos dentro del Plan Básico de Ordenamiento Territorial (Рвот), para garantizar su protección, uso y manejo.

- Que el Municipio eleve consulta a la Agencia Nacional Minera sobre los títulos mineros presentes en el territorio, con el fin de verificar su estado y vigencia, ya que no todos los títulos mineros otorgados en Colombia están priorizados para su exploración y explotación. 
- Es necesario que el Consejo de Planeación Territorial, como organismo de consulta ciudadana, pueda ejercer sus funciones adecuadamente y no sea sometido a su desconocimiento o marginación de los procesos de planificación. De esta manera, se garantizaría una de las formas de participación efectiva de la sociedad civil en la formulación del Plan de Desarrollo como de la actualización del Plan Básico de Ordenamiento Territorial (Рвот), lo cual posibilita el debido proceso en la adopción de estos instrumentos de planificación.

\section{Referencias}

Agencia Nacional de Minería. (2019). Catastro Minero de Colombia. Recuperado de http://www.cmc. gov.co:8080/CmcFrontEnd/consulta/index.cmc

Andrade Pérez, G. I., \& Corzo Mora, G. A. (2011). ¿Qué y dónde conservar? Bogotá, Colombia: Parques Nacionales Naturales de Colombia. Recuperado de https://bit.ly/3jvn746

Colombia. Acuerdo N. ${ }^{\circ} 010$ de 2016. Concejo Municipal de Rioblanco, Tolima. Por el cual se adopta el Plan de Desarrollo Todos somos Rioblanco 2016-2019. Recuperado de http://www.rioblancotolima.gov.co/planes/plan-de-desarrollo-20162019

Colombia. Decreto 3600 de 2007. Presidencia de la República de Colombia. Por el cual se reglamentan las disposiciones de las Leyes 99 de 1993 y 388 de 1997 relativas a las determinantes de ordenamiento del suelo rural y al desarrollo de actuaciones urbanísticas de parcelación y edificación en este tipo de suelo y se adoptan otras disposiciones. Diario oficial, n. 46757.

Colombia. Ley 1382 de 2010. Por el cual se modifica la Ley 685 de 2001 Código de Minas. Diario oficial, n. ${ }^{\circ} 47618$.

Colombia. Ley 685 de 2001. Por la cual se expide el Código de Minas y se dictan otras disposiciones. Diario oficial, n. ${ }^{\circ} 44545$.

Colombia. Rioblanco Tolima. Plan Básico de Ordenamiento Territorial. 2000.

Colombia. Ley 1454 de 2011. Ley Orgánica de Ordenamiento Territorial (LOOT). Por el cual se dictan normas orgánicas sobre ordenamiento territorial y se modifican otras disposiciones.

Colombia. Ley 388 de 1997. Por la cual se modifica la Ley 9 de 1989, y la Ley 2 de 1991 y se dictan otras disposiciones.

Colombia. Ley 152 de 1994. Por la cual se establece la Ley Orgánica del Plan de Desarrollo.

Constitución Política de Colombia de 1991. Artículos 8, 58, 79, 80, 334, 360. Publicada en la Gaceta Constitucional n. ${ }^{\circ} 116$ de 20 de julio de 1991.

Convención de Ramsar y Grupo de Contacto eHAA. (2008). Uso racional de los humedales. Recuperado de http://www.ramsar.org/es/acerca-de/uso-racional-de-los-humedales

Fierro Morales, J. (2012). Políticas mineras en Colombia. Bogotá, Colombia: Instituto para una Sociedad y un Derecho Alternativo (ILSA). Recuperado de https://bit.ly/3lOMb9p 
Martínez, P. A., \& González, C. H. (2020). La gestión ambiental en el ordenamiento territorial para la conservación de los servicios ambientales en Rioblanco Tolima (Tesis de Maestría en Desarrollo Sostenible y Medio Ambiente). Manizales, Colombia: Universidad de Manizales.

Paruelo, J. M., Laterra, P, \& Viglizzo, E. F. (2015). Plan operativo para incorporar los servicios ecosistémicos en el ordenamiento territorial. En Paruelo, J. M., Jobbágy, E. G., Laterra, P. Dieguez, H., García Collazo, M. A., Panizza, A. (edit.) Ordenamiento Territorial Rural. Conceptos, métodos y experiencias (pp.159-172). Recuperado de https://www.researchgate.net/publication/302742389

Rudas, G. (2010). Política ambiental del presidente Uribe, 2002-2010. Niveles de prioridad y retos futuros. Bogotá, Colombia: Informe del Consejo Nacional de Planeación.

Van der Hammen, T., \& Andrade, G. (2003). Estructura ecológica principal de Colombia. Bogotá, Colombia: IDEAM.

\section{Bibliografía recomendada}

Acevedo Ortiz, D. A., \& Flórez Yepes, G. (2016). Estudio de los instrumentos normativos de ordenamiento ambiental del municipio de Turbo (Antioquia), en el marco de las políticas de ordenamiento ambiental de Colombia. Revista Luna Azul, (42), 167-184. Recuperado de https://www.redalyc.org/ articulo.oa?id=321744162009

Colombia. Ley 99 de 1993. Por la cual se crea el Ministerio del Medio Ambiente, se reordena el Sector Público encargado de la gestión y conservación del medio ambiente y los recursos naturales renovables, se organiza el Sistema Nacional Ambiental, sinA, y se dictan otras disposiciones.

Montes Lira, P. F. (2001). El ordenamiento territorial como opción de políticas urbanas y regionales en América Latina y el Caribe (Serie medio ambiente y desarrollo n. $\left.{ }^{\circ} 45\right)$. Recuperado del sitio de CEPAL http://hdl.handle.net/11362/5739

Noriega Moreno, L. H. (2018). La realidad de la planificación territorial en Colombia en el contexto de la relación entre la población y la estructura ecológica principal. El conflicto por la apropiación del suelo. Revista Internacional de Dereito Ambiental, 7 (20), 199-236. 